\title{
Phase i study of 'dose-dense' pemetrexed plus carboplatin/radiotherapy for locally advanced non-small cell lung carcinoma
}

Xinglei Shen ${ }^{1}$, Albert DeNittis ${ }^{2}$, Maria Werner-Wasik ${ }^{1}$, Rita Axelrod ${ }^{3}$, Paul Gilman ${ }^{4}$, Thomas Meyer ${ }^{4}$, Joseph Treat ${ }^{5}$, Walter J Curran ${ }^{1,6}$, Mitchell Machtay ${ }^{1,7^{*}}$

\begin{abstract}
Background: This phase I study investigates the feasibility of carboplatin plus dose-dense (q2-week) pemetrexed given concurrently with radiotherapy (XRT) for locally advanced and oligometastatic non-small cell lung cancer (NSCLC).

Methods: Eligible patients had Stage III or IV (oligometastatic) NSCLC. Patients received XRT to 63 Gy in standard fractionation. Patients received concurrent carboplatin (AUC $=6)$ during weeks 1 and 5 of XRT, and pemetrexed during weeks 1, 3, 5, and 7 of XRT. The starting dose level (level 1) of pemetrexed was $300 \mathrm{mg} / \mathrm{m}^{2}$. Following the finding of dose limiting toxicity (DLT) in dose level 1, an amended dose level (level 1A) continued pemetrexed at $300 \mathrm{mg} / \mathrm{m}^{2}$, but with involved field radiation instead of extended nodal irradiation. Consolidation consisted of carboplatin $(A \cup C=6)$ and pemetrexed $\left(500 \mathrm{mg} / \mathrm{m}^{2}\right)$ q3 weeks $\times 2-3$ cycles.

Results: Eighteen patients were enrolled. Fourteen patients are evaluable for toxicity analysis. Of the initial 6 patients treated on dose level 1, two experienced DLTs (one grade 4 sepsis, one prolonged grade 3 esophagitis). There was one DLT (grade 5 pneumonitis) in the 8 patients treated on dose level 1A. In 16 patients evaluable for response (4 with oligometastatic stage IV disease and 12 with stage III disease), the median follow-up time is 17.8 months. Thirteen of 16 patients had in field local regional response. The actuarial median survival time was 28.6 months in all patients and 34.7 months (estimated) in stage III patients.
\end{abstract}

Conclusions: Concurrent carboplatin with dose-dense (q2week) pemetrexed at $300 \mathrm{mg} / \mathrm{m}^{2}$ with involved field XRT is feasible and encouraging in patients with locally advanced and oligometastatic NSCLC.

Trial Registration: ClinicalTrials.gov NCT00330044

\section{Background}

Concurrent chemoradiation has been established as the standard of care for non-operable stage III non-small cell lung cancer (NSCLC) [1-4]. With this approach, the median survival time is approximately 17 months and about $15 \%$ of patients survive $5+$ years. Concurrent combined modality therapy has improved survival over single modality or sequential therapy [1-4], but overall outcomes remain poor.

The optimal chemotherapy regimen to use with concurrent radiation therapy remains uncertain. Initial

\footnotetext{
*Correspondence: mitchell.machtay@uhhospitals.org 'Department of Radiation Oncology, Kimmel Cancer Center, Jefferson Medical College of Thomas Jefferson University, Philadelphia, USA Full list of author information is available at the end of the article
}

studies of concurrent treatment have used cisplatin plus a second drug given at near-systemic doses for two cycles during RT [1-4]. No platinum based doublet has clear proven superiority over other regimens. These combinations have significant toxicity with high rates of esophagitis, nausea/vomiting, and myelosuppression.

Alternative less toxic chemotherapy drugs and schedules, most notably weekly carboplatin/paclitaxel regimens have been extensively studied [5-8]. This regimen has been criticized because safe and feasible "radiosensitizing" doses of carboplatin (AUC $=1.5$ to 2) and paclitaxel $(45-50 \mathrm{mg} / \mathrm{m} 2)$ are well below the dose intensities considered independently active

\section{Biomed Central}


against NSCLC[9]. Thus, while this regimen may have excellent radiosensitization properties, it likely has little effect on tumor populations outside of the radiation portal. The solution has been to combine concurrent chemoradiotherapy with induction $[8,10]$ or consolidation $[4,5]$ chemotherapy given at systemic doses. This approach necessarily delays the initiation of either local or systemic therapy.

In contrast, the new cytotoxic drug pemetrexed has independent activity against NSCLC and reduced toxicity $[11,12]$, and may be feasible to deliver at nearsystemic doses with concurrent radiotherapy [13]. Pemetrexed belongs to the antimetabolite class of antineoplastic drugs. It targets multiple molecules within the folate metabolism pathway, including thymidylate synthase and dihydrofolate reductase. Preclinical data support the hypothesis that pemetrexed serves as a radiosensitizer in addition to having independent activity against NSCLC in vitro and in vivo $[14,15]$. A large randomized trial comparing pemetrexed to docetaxel in second line treatment of metastatic NSCLC showed similar response and survival with a more favorable toxicity profile for pemetrexed [16]. In the first line chemotherapy setting for advanced NSCLC, a randomized trial showed that pemetrexed in combination with cisplatin resulted in equivalent survival to gemcitabine with cisplatin [17].

Preliminary results of several studies testing pemetrexed plus radiotherapy have been presented. A phase I trial showed that pemetrexed at a dose of $500 \mathrm{mg} / \mathrm{m} 2$ q3weeks (Weeks 1, 4, and 7) could be combined with a full course of standard radiotherapy [13]. Recent update from the CALBG trial \#30407 showed that systemic dose pemetrexed may be combined with systemic dose carboplatin (AUC $=6$ ) q3weeks with concurrent radiation to 70 Gy with acceptable toxicities. Efficacy data presented at ASCO 2009 is encouraging, with a median survival time of 22.3 months [18].

The modest toxicity profile of pemetrexed led us to consider whether further intensification of pemetrexed during chemoradiotherapy could be accomplished, with the long-term goal of improving local and distant control. This strategy has successfully improved outcomes in node-positive breast cancer $[19,20]$, aggressive nonHodgkin's lymphoma [21], and ovarian cancer[22]. We designed and initiated a pilot (phase I) feasibility trial of dose-dense (q2-week) pemetrexed with systemic dose carboplatin and concurrent radiotherapy in the treatment of locally advanced and oligometastatic NSCLC.

\section{Methods}

This was a prospective, investigator-initiated clinical trial, approved by the scientific Clinical Research Committee of the Kimmel Cancer Center at Thomas
Jefferson University as well as the Internal Review Board (IRB) of Thomas Jefferson University (TJU). The study was also approved by the IRB of the participating medical center, Lankeanu Hospital (Lower Merion, PA), a member of the Jefferson Health System. Eli Lilly Inc. supported the study with a grant to Thomas Jefferson University; however, the study was written, conducted and analyzed by TJU and Lankenau investigators and sponsored by TJU, independently from any corporate entity. The study was monitored by the Kimmel Cancer Center's Data and Safety Monitoring Board in addition to the investigators.

\section{Patients/Eligibility}

This study was available to patients with locally advanced NSCLC who required definitive full dose radiotherapy as part of their treatment plan. This included stage IIIA, IIIB and oligometastatic stage IV (without diffuse hematogenous metastases) NSCLC. Patients with stage IV NSCLC were only eligible if they had bulky local-regional disease deemed to require high dose local radiotherapy and no symptoms from their extrathoracic disease.

Other eligibility requirements included Zubrod performance status $0-1$, absence of severe $(>10 \%)$ weight loss, FEV1 $>1000 \mathrm{cc}$, serum creatinine $<1.5 \mathrm{mg} / \mathrm{dl}$, serum bilirubin $<1.5 \mathrm{mg} / \mathrm{dl}$, SGOT $<1.5$ times institutional upper limits of normal, hemoglobin $>8.0 \mathrm{~g} / \mathrm{dl}$, ANC $>2000$ cells $/ \mathrm{mm}^{3}$, platelets $>100,000$ cells $/ \mathrm{mm}^{3}$, and no recent ( $<6$ months) myocardial infarction, unstable angina, congestive heart failure or uncontrolled arrhythmia. Exclusion criteria also included prior chemotherapy for lung cancer and/or prior thoracic radiotherapy that would result in field overlap.

\section{Radiotherapy}

Radiotherapy (RT) planning via 3-dimensional, CT-scan based planning was required. Intensity modulated radiation therapy (IMRT) was not used. The choice of field arrangements was left to the discretion of the radiation oncologist, and typically consisted of two to four conformally planned, coplanar fields designed to minimize irradiation of the spinal cord and contralateral lung. Respiratory gating was not used. The protocol-specified dose of radiotherapy to tumor as defined by CT (and PET scan where appropriate) was 63 Gy, given in conventional (1.8-2 Gy) once daily fractionation.

The initial protocol design (Dose Level \#1) was to irradiate a large volume to $45 \mathrm{~Gy}$, followed by a conedown to the gross tumor (plus a small margin) for an 18 Gy boost. This initial volume would include the gross tumor plus a generous margin (at least $2 \mathrm{~cm}$ ) and the comprehensive bilateral mediastinal nodal space (from the thoracic inlet to at least $5 \mathrm{~cm}$ below the 
carina). In some cases, radiotherapy fields included the inferior mediastinal nodes to the crus of the diaphragm (if subcarinal nodes were involved) and/or contralateral hilar nodes (if bilateral mediastinal nodes were involved), based on the principle of irradiating at least one echelon of lymph nodes beyond that known to be grossly involved.

The protocol was subsequently amended in response to two DLTs to require the use of involved field irradiation from the start of radiotherapy (Dose Level \#1A). Involved field radiotherapy included areas positive by CT scan and/or PET scan, with an option to include areas located geographically between two involved areas (e.g. inclusion of the ipsilateral hilum if the adjacent mediastinal nodes are involved). Contralateral mediastinal, contralateral hilar and supraclavicular nodes were no longer electively irradiated.

\section{Chemotherapy/Dose Escalation Plan}

The study was designed to use a fixed dose of carboplatin (AUC $=6$, based upon the Cockroft-Gault formula), during Weeks 1 and 5 of radiotherapy (preferably during Day 1 or 2 of those weeks). There were no plans to alter this dose/schedule.

The pemetrexed design of the study was to administer this drug on a biweekly basis (Weeks 1, 3, 5, and 7) during radiotherapy; on Weeks 1 and 5 it would be given together on the same day with carboplatin. The starting dose of pemetrexed for the study was $300 \mathrm{mg} / \mathrm{m}^{2}$, with plans to dose escalate to 400 and $500 \mathrm{mg} / \mathrm{m}^{2}$ in subsequent patients based upon analysis of feasibility and toxicity (these dose escalations did not successfully occur).

Dose/schedule modifications were allowed for toxicity. If grade 3-4 neutropenia/thrombocytopenia and/or grade 3 non-hematologic toxicity occurred, all agents (RT, carboplatin, radiotherapy, and pemetrexed) were to be held for 1-2 weeks. When toxicity resolved to Grade $0-1$, treatment was to be resumed with a reduction in pemetrexed by $50 \mathrm{mg} / \mathrm{m} 2$ (i.e. from $300 \mathrm{mg} / \mathrm{m} 2$ to $250 \mathrm{mg} / \mathrm{m} 2$ ). If a second episode of Grade 4 hematologic or grade 3 non-hematologic toxicity were to occur, this would be considered a DLT and the patient removed from study.

Patients who successfully completed carboplatin/ pemetrexed and concurrent radiotherapy were allowed to continue on to consolidation carboplatin/pemetrexed after recovering from acute effects of chemoradiotherapy. The consolidation regimen consisted of 2-3 cycles of carboplatin $($ AUC $=6)$ and pemetrexed $\left(500 \mathrm{mg} / \mathrm{m}^{2}\right)$ q3 weeks. Growth factor (G-CSF or GM-CSF) support was recommended.

Patients were given a subcutaneous injection of B12 $(1000 \mathrm{mcg})$ before starting study treatment and once per month while on study. Folic acid (1000 mcg daily) was also prescribed starting Day 1.

\section{Study Endpoints/Analysis plan}

The primary endpoint of the study was dose-limiting toxicity (DLT), defined as any one of the following serious adverse events (SAE's) as determined by the study investigators and medical monitor to be the result of study treatment:

1. Death within 30 days after the completion of radiotherapy or within 90 days of start of radiotherapy.

2. Grade 4 non-hematologic toxicity occurring during or within 30 days after the completion of radiotherapy or within 90 days of start of radiotherapy.

3. Grade 3 pulmonary toxicity within 90 days after the completion of chemoradiotherapy.

4. Prolonged ( $>14$ days) grade 3 esophagitis 30 days after the completion of radiotherapy or within 90 days of start of radiotherapy preventing the patient from being able to proceed with anti-cancer treatment.

5. Inability to complete at least 54 Gy of thoracic radiotherapy due to toxicity

The Kimmel Cancer Center of Thomas Jefferson University assigned an independent medical monitor to review SAE's with the study investigators and help determine if/when a DLT occurred and if pemetrexed dose may be escalated (or de-escalated) per study protocol.

The statistical plan called for dose escalation from Dose Level \#1 (300 mg/m2) to Dose Level \#2 (400 mg/ $\mathrm{m} 2$ ) if/when none of the first three or one of the first six patients enrolled and evaluable experienced a DLT. A similar plan was made for further escalation beyond Dose Level \#2. If at any given dose level, a second DLT occurred, the study was to be closed to further accrual, discussed with the medical monitor and IRB, and modified in order to assure patient safety.

Secondary endpoints included local tumor response rates, and progression-free and overall survival. Survival times were calculated from date of registration on trial.

\section{Results}

\section{Accrual/Feasibility}

This study enrolled its first patient in April 2006; the final patient enrolled in April 2008. There were several time periods where the study was closed for safety/toxicity assessment.

Of the 18 patients accrued, two patients were never treated with study chemoradiotherapy and are not evaluable for any study endpoints. Both patients were found to be fully eligible, signed informed consent, and 
were enrolled using our institution's registration mechanism. One patient withdrew consent and switched to non-protocol chemoradiotherapy; the second developed a GI bleed and severe anemia prior to receiving any study medication.

Of the remaining 16 patients, two patients are not evaluable for the primary study endpoint (determination of DLT). These two patients (Pts 9 and 10) were enrolled onto Dose Level \#2 (400 mg/m2 pemetrexed), based upon what initially appeared to be a favorable toxicity profile among the first six evaluable patients in Dose Level \#1. However, as patients \#9 and 10 were beginning treatment, a delayed DLT in Dose Level \#1 occurred (prolonged esophagitis/weight loss requiring feeding tube). After discussions among the investigators and medical monitor, they were offered the option of withdrawing or continuing with a reduced pemetrexed dose of $250 \mathrm{mg} / \mathrm{m} 2$ (Dose Level \# -1). These patients opted to continue at the reduced pemetrexed dose. Of note, these two patients completed therapy on schedule with no significant non-hematologic toxicities.

\section{Patient characteristics}

Patient characteristics are shown in Table 1. The median patient age was 70 . No patient was considered a candidate for surgical resection. The median FEV1 was 2.00 L. Most patients had stage III disease (6 stage IIIA; 6 stage IIIB) and 4 patients had oligometastatic (3 brain, 1 bone) stage IV disease. Histology was squamous in 3 patients, adenocarcinoma in 9 patients, and nototherwise-specified (NOS) in 4 patients.

\section{Treatment compliance}

Fourteen patients were evaluable for the study's primary endpoints of feasibility and assessment of DLT; all these patients received both doses of carboplatin $(\mathrm{AUC}=6)$, and 13 of 14 patients completed their full course of radiotherapy. All 14 patients received at least 3 of four planned courses of pemetrexed ( 7 of 14 received all four courses; the other 7 missed one course due to neutropenia and/or anemia). Of the 13 patients who completed radiotherapy, 8 received at least two cycles of adjuvant chemotherapy and 5 did not ( 2 developed progressive disease, 2 had dose limiting toxicities precluding further therapy, and 1 developed a pulmonary embolism).

\section{Toxicity}

Of the six patients enrolled into Dose Level \#1, two patients developed DLT. One DLT was neutropenic fever, sepsis and multi-organ failure, which appeared to arise in the setting of colonic perforation at the site of chronic diverticulitis. The patient recovered after urgent surgery and a prolonged hospital course, but was unable to resume any anti-cancer treatment. The second DLT was a delayed and prolonged grade 3 esophagitis after completion of concurrent chemoradiation requiring feeding tube placement, preventing the patient from receiving any additional anticancer treatment. Based on these two DLTs, Dose Level \#1 was considered infeasible as originally designed and the study was amended utilizing reduced radiotherapy fields.

Dose Level \#1A enrolled eight patients evaluable for toxicity. One patient suffered a DLT: an 83 year-old man with significant underlying COPD developed

Table 1 Patient Characteristics and Treatment Delivery

\begin{tabular}{|c|c|c|c|c|c|c|}
\hline Patient & $\underline{\text { Age }}$ & $\underline{\text { Stage }}$ & Histology & Dose Level & $\underline{\text { XRT dose/Tx time (Gy/days) }}$ & Concurrent Pemetrexed Dose Received $\left(\mathrm{mg} / \mathrm{m}^{2}\right)$ \\
\hline $1 \mathrm{TJ}$ & 55 & $\| \mathrm{IIB}$ & $\mathrm{Ad}$ & 1 & $63 / 45$ & 1150 \\
\hline 2 ST & 72 & $\| I \mathrm{~B}$ & $\mathrm{Sq}$ & 1 & $63 / 48$ & 900 \\
\hline $3 \mathrm{LM}$ & 64 & IIIA & $\mathrm{Ad}$ & 1 & $39.6 / 33^{*}$ & 900 \\
\hline$\underline{4 \mathrm{SR}}$ & 45 & IIIB & $\mathrm{Ad}$ & 1 & $59.4 / 57$ & 1200 \\
\hline$\underline{\mathrm{MW}}$ & 54 & IV & $\mathrm{Ad}$ & 1 & $63 / 47$ & 1200 \\
\hline $6 \mathrm{SR}$ & 75 & IIIA & $\mathrm{Ad}$ & 1 & $63 / 51$ & 900 \\
\hline $7 \mathrm{PH}$ & 68 & IIIB & NOS & $2 /-1$ & $63 / 55$ & 1300 \\
\hline $8 \mathrm{JD}$ & 72 & $\| I \mid B$ & $\mathrm{Ad}$ & $2 /-1$ & $63 / 51$ & 1150 \\
\hline $9 \mathrm{JB}$ & 78 & IIIB & NOS & $1 \mathrm{~A}$ & $63 / 47$ & 900 \\
\hline $10 \mathrm{BS}$ & 61 & IV & NOS & $1 \mathrm{~A}$ & $63 / 49$ & 1200 \\
\hline $11 \mathrm{ES}$ & 63 & IV & $\mathrm{Ad}$ & $1 \mathrm{~A}$ & $63 / 48$ & 900 \\
\hline $12 \mathrm{JB}$ & 83 & IIIA & $\mathrm{Sq}$ & $1 \mathrm{~A}$ & $63 / 53$ & 1200 \\
\hline $13 \mathrm{EH}$ & 73 & IV & $\mathrm{Ad}$ & $1 \mathrm{~A}$ & $63 / 54$ & 1200 \\
\hline $14 \mathrm{ML}$ & 74 & IIIA & $\mathrm{Ad}$ & $1 \mathrm{~A}$ & $63 / 47$ & 1200 \\
\hline $15 \mathrm{CN}$ & 74 & IIIA & $\mathrm{Sq}$ & $1 \mathrm{~A}$ & $63 / 49$ & 1200 \\
\hline 16 UG & 62 & IIIA & NOS & $1 \mathrm{~A}$ & $63 / 48$ & 1200 \\
\hline
\end{tabular}

*Pt suffered neutropenic fever/sepsis requiring treatment discontinuation.

Histology: $\mathrm{Ad}=$ Adenocarcinoma; $\mathrm{Sq}=$ Squamous; $\mathrm{NOS}=$ Not-otherwise-specified. 
respiratory decompensation one week after completing chemoradiotherapy, and ultimately died. The treating physicians initially determined that this was 'unrelated' to study treatment and primarily related to age and underlying respiratory insufficiency. However, on comprehensive review by the entire study investigation team and independent medical monitors, it was concluded that the patient's death should be considered 'possibly related' to treatment.

The overall toxicity profile of sixteen evaluable patients is shown in Table 2 . The most common toxicity was neutropenia, including 7 cases of grade 3-4 neutropenia. There was one Grade 5 toxicity and one patient with Grade 4 non-hematologic toxicity (neutropenic fever/sepsis). Three patients developed grade 3 esophagitis. One patient developed grade 3-4 fatigue. In Dose Level \#1, the rate of any Grade 3 or greater non-hematologic toxicity was 33\% (2/6); in Dose Level \#1A, the rate of Grade 3 or greater non-hematologic toxicity was $25 \%(2 / 8)$.

\section{Response/Efficacy}

A total of 15 patients are evaluable for treatment response and patterns of failure (Table 3). In-field localregional tumor response (partial or complete response) by RECIST criteria was observed in 14, while the other patient demonstrated stable disease without evidence of in-field progression on serial CT and/or PET scans at 40 months. Three patients developed local-regional recurrence as the first site of failure. One progressed just outside of the radiotherapy portal ('marginal miss') and a second patient progressed in an elective nodal region not treated with involved field radiation. These two patients, each with a small focus of intrathoracic progression, were treated with additional radiotherapy. A third patient who progressed within the treatment field at 22 months was treated with salvage brachytherapy. In all, five patients progressed locally at a median time of 10 months.

Three patients (20\%) without distant metastases at registration developed distant metastases as the first site of failure at a median time of 5 months. Four additional patients $(27 \%)$ had oligometastatic disease at presentation, and all four succumbed to progressive systemic metastases at a median time of 6 months.

There have been nine deaths among the 16 study patients, seven from metastatic NSCLC, one from respiratory failure (due to intercurrent disease and/or treatment complication) and one from an unrelated myocardial infarction. The median follow-up period for all patients is 15.2 months ( $3-40$ months) and 24.2 months for surviving patients (12-40 months). The one-year actuarial overall survival rate is $63 \%$, and two-year $56 \%$. Median survival time for all patients was 28.6 months, and among stage III patients it was not reached, but
Table 2 Acute Toxicity

\begin{tabular}{|c|c|c|c|c|}
\hline Toxicity & $\begin{array}{l}\text { Grade 1- } \\
2\end{array}$ & $\begin{array}{l}\text { Grade } \\
3\end{array}$ & $\begin{array}{l}\text { Grade } \\
4\end{array}$ & $\begin{array}{l}\text { Grade } \\
5\end{array}$ \\
\hline \multicolumn{5}{|c|}{ Dose Level $\# 1 *(N=6)$} \\
\hline Neutropenia & 1 & 2 & $1^{t+}$ & - \\
\hline Thrombocytopenia & 1 & 1 & $1^{t+}$ & - \\
\hline Anemia & 3 & 1 & - & - \\
\hline Esophagitis & 3 & 1 & - & - \\
\hline Pneumonitis & 2 & 1 & - & - \\
\hline Sepsis & - & - & $1^{t+}$ & - \\
\hline Fatigue & 4 & - & - & - \\
\hline Elevated LFT's & 4 & $1^{+\dagger}$ & - & - \\
\hline Elevated Creatinine & 1 & - & - & - \\
\hline Worst Toxicity Overall & 2 & 3 & 1 & - \\
\hline $\begin{array}{l}\text { Worst non-heme Toxicity } \\
\text { Overall }\end{array}$ & 4 & 1 & 1 & - \\
\hline
\end{tabular}

\begin{tabular}{lcccc}
\hline \multicolumn{5}{c}{ Dose Level \#1A* $\mathbf{N}=\mathbf{8})$} \\
\hline Neutropenia & 4 & 3 & 1 & - \\
\hline Thrombocytopenia & 2 & 1 & - & - \\
\hline Anemia & 6 & 1 & - & - \\
\hline Esophagitis & 5 & 2 & - & - \\
\hline Pneumonitis & 3 & - & - & $11^{\S}$ \\
\hline Sepsis & - & - & - & - \\
\hline Fatigue & 5 & - & $1{ }^{\S}$ & - \\
\hline Elevated LFT's & 1 & - & - & - \\
\hline Elevated Creatinine & 1 & - & - & - \\
\hline Worst Toxicity Overall & 3 & 3 & - & 1 \\
\hline Worst non-heme Toxicity & 4 & 1 & - & 1 \\
Overall & & & & \\
\hline
\end{tabular}

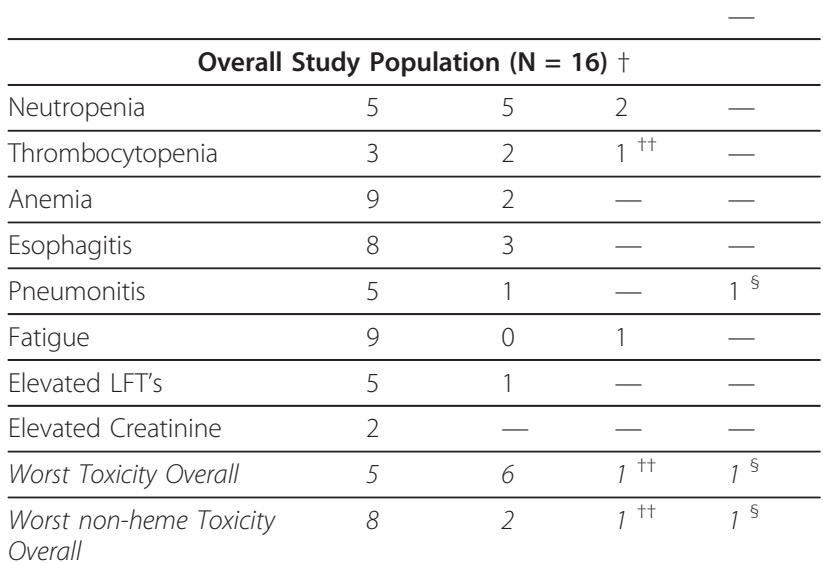

Overall

* Dose Level \#1 and Dose Level \#1A used the same doses (pemetrexed 300 $\mathrm{mg} / \mathrm{m} 2$ q2-week); however Dose Level \#1 used extended field RT and Dose Level \#1A used involved field RT.

† Overall study population included all of the patients treated in Dose Level \#1 and Dose Level \#1A, as well as two patients who were re-assigned from Dose Level \#2 (400 mg/m2) to Dose Level -I $(250 \mathrm{mg} / \mathrm{m} 2)$ because of safety purposes. t† Single patient with sepsis and multi-organ failure in the setting of acute diverticulitis.

$\S$ Elderly (83-year old) patient with COPD, died from respiratory failure 30 days post-RT. 
Table 3 Treatment Response and Outcomes Data

\begin{tabular}{|c|c|c|c|c|}
\hline Patient & Dose Level & Initial Local response & Distant Metastases & Survival Status \\
\hline $1 \mathrm{TJ}$ & 1 & $\mathrm{NR} / \mathrm{SD}$ & None & AWD @ 40 mo. \\
\hline $2 \mathrm{ST}$ & 1 & $P R$ & Diffuse Mets & DOD @ 34 mo. \\
\hline $3 \mathrm{LM}$ & 1 & $P R$ & Diffuse Mets & DOD@ @ 15 mo. \\
\hline $4 \mathrm{SR}$ & 1 & $C R$ & Diffuse Mets & DOD @ 29 mo. \\
\hline $5 \mathrm{MW}$ & 1 & $P R$ & Mets at presentation & DOD@6 mo. \\
\hline $6 \mathrm{SR}$ & 1 & $C R$ & None & NED @ 26 mo. \\
\hline $7 \mathrm{PH}$ & $2 /-1$ & $C R$ & None & AWD@26 mo. \\
\hline $8 \mathrm{JD}$ & $2 /-1$ & $P D^{*}$ & None & NED @ 27 mo. \\
\hline $9 \mathrm{JB}$ & $1 \mathrm{~A}$ & $C R$ & None & NED @ 24 mo. \\
\hline $10 \mathrm{BS}$ & $1 \mathrm{~A}$ & $P R$ & Mets at presentation & DOD@ @ mo. \\
\hline $11 \mathrm{ES}$ & $1 \mathrm{~A}$ & $P R$ & Mets at presentation & DOD@6 mo. \\
\hline $12 \mathrm{JB}$ & $1 \mathrm{~A}$ & NA & NA & DID/DOC@ @ mo. \\
\hline $13 \mathrm{EH}$ & $1 \mathrm{~A}$ & $P R$ & Mets at presentation & DOD@ 6 mo. \\
\hline $14 \mathrm{ML}$ & $1 \mathrm{~A}$ & $P R$ & None & NCRM @ 9 mo. \\
\hline $15 \mathrm{CN}$ & $1 \mathrm{~A}$ & $P R$ & None & AWD@18 mo. \\
\hline 16 UG & $1 \mathrm{~A}$ & $C R$ & None & AWD @ 18 mo. \\
\hline
\end{tabular}

NR/SD: No Response/Stable Disease.

PR: Partial Response.

CR: Complete Response.

PD*: Progressive Disease (one patient had PD just outside of the radiotherapy field edge).

NA: Not Assessable - one patient is not assessable or evaluable due to early (possibly treatment related) death.

AWD: Alive with Disease

DOD: Died of Disease.

NED: No Evidence of (Active) Disease.

DID/DOC: Died of intercurrent Disease and/or treatment Complications.

NCRM: Non-cancer related mortality (one patient died of a MI while NED).

estimated at 34.7 months (Figure 1). Median survival time for oligometastatic patients was 6 months.

\section{Discussion}

We demonstrate the feasibility and safety of combining dose-dense (q2-week) pemetrexed and systemic dose

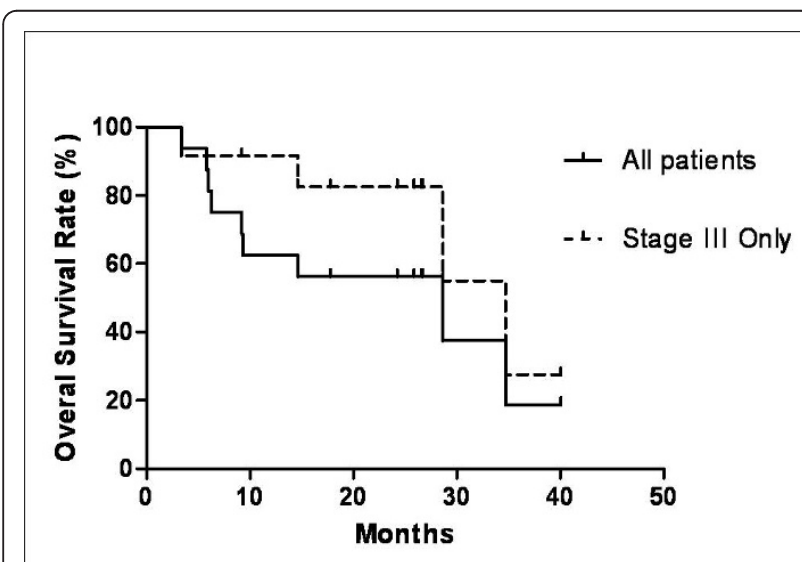

Figure 1 Kaplan-Meier survival curve. Median survival time for all patients treated is 28.6 months. For stage III patients, the median survival time was not reached, but estimated at 34.7 months. carboplatin (AUC $=6$ ) with radiotherapy for NSCLC. All patients were able to receive both doses of carboplatin and at least three (out of four) doses of pemetrexed with concurrent radiation. All but one patient was able to complete radiotherapy. The main toxicity observed in this study was myelosuppression, primarily manifesting as neutropenia.

Toxicity was decreased by amending the study to mandate the use of smaller (involved-field) radiotherapy treatment plans. We found that 2 of the first 6 patients enrolled in our study suffered non-hematologic DLT's when treated with comprehensive mediastinal irradiation. After changing the radiotherapy planning portion of our study, non-hematologic DLT's occurred in 1 of 8 patients. This patient was an 83 year-old man with severe COPD who died from respiratory decompensation following completion of chemoradiotherapy. We conservatively report this as a DLT although intercurrent disease played a significant role.

When the study was originally designed (in 2005), standard RT consisted of comprehensive mediastinal irradiation to 45-50 Gy followed by a boost to gross tumor to 60-64 Gy, as used in the Radiation Therapy Oncology Group (RTOG) clinical trials [2,23,24]. 
More recently, data show that outcomes (local control and survival) are not compromised by using involvedfield radiotherapy from the start of treatment, and reduced treatment volume may allow the delivery of higher radiotherapy dose $[25,26]$.

In contrast to previous chemoradiation regimens containing reduced radiosensitizing doses of carboplatin and paclitaxel, this study investigated the used of systemic doses of carboplatin (AUC $=6)$, which has independent activity in NSCLC. The dose dense pemetrexed at $300 \mathrm{mg} / \mathrm{m}^{2}$ also approaches systemic dose of pemetrexed. Although our study was not powered for assessment of anti-tumor efficacy, the results of our survival data are very promising with a median survival time of 28.6 months in all patients, and estimated 34.7 months in stage III patients.

We note that almost all evaluable patients had a clinical local response and at least short term in-field local control of their cancer. Although pathologic assessment of local disease was not performed in our study, our data do support the hypothesis that dose-dense carboplatin/pemetrexed is an effective radiosensitization regimen for definitive therapy of locally advanced nonoperative NSCLC.

Despite excellent in field control, progression of disease outside the radiation treatment fields remains exceedingly common. Our study included four patients with oligometastatic diease, and each of these patients quickly progressed systemically with a median survival of only 6 months. Three additional patients developed metastatic disease as the first site of recurrence, and two patients recurred locoregionally outside the treatment field. We hypothesize that this reflects chemoresistance in micrometastatic deposits outside of the radiotherapy fields. With improving local control, the ability to control micrometastatic disease has increasing importance in improving overall survival. It is possible that higher dose intensity of platinum, pemetrexed and/or addition of a third cytotoxic drug could be more effective, although at a cost of higher toxicity.

A recent study by Cullen et al. failed to show an advantage to increased dose intensity of pemetrexed in advanced/metastatic (platinum-refractory) NSCLC[27]. This study compared $500 \mathrm{mg} / \mathrm{m} 2$ q3week versus $900 \mathrm{mg} / \mathrm{m} 2$ q3week. It is unclear whether dose intensification of pemetrexed using a q2week schedule, or in a less heavily pre-treated population such as ours, might yield different results.

Another strategy might be to add a biologic agent such as a vascular targeting drug or an anti-EGFR agent to our regimen. The Cancer and Leukemia Group B (CALGB) study \#30407 investigated in a prospective phase II randomized trial combining carboplatin/radiotherapy and pemetrexed (standard $500 \mathrm{mg} / \mathrm{m} 2 \mathrm{q} 3$-week schedule) with or without cetuximab. Presented in abstract form at the 2009 ASCO national meeting, the carboplatin/pemetrexed/RT arm had a promising median survival time of 22.3 months, but the addition of cetuximab did not result in improved survival with a median survival time of 18.7 months [18].

A complementary strategy may be more careful selection of semi-customized treatments. Randomized studies of single agent pemetrexed in second line chemotherapy treatment of NSCLC and of platinum/pemetrexed in first line treatment of advanced NSCLC demonstrated an improved survival in patients with non-squamous NSCLC, and a worse outcome in patients with squamous histology [16,17]. This difference may be related to increased expression of thymidylate synthase (TS) in squamous cancers or other proteins relevant to the target of pemetrexed[17]. Our study did not collect tissue to perform this analysis, but evaluation of TS will be important to future studies of pemetrexed and radiation in NSCLC.

\section{Conclusions}

Dose-dense (q-2week) pemetrexed at a dose of $300 \mathrm{mg} /$ $\mathrm{m} 2$ and carboplatin $(\mathrm{AUC}=6)$ combined with concurrent involved field radiation is feasible. It was not feasible with extended field radiotherapy. Responses are encouraging and this is a suitable platform for further development of future combined modality trials.

\section{Acknowledgements \\ This study was partially supported by a grant from Eli Lilly, Inc.}

\section{Author details}

${ }^{1}$ Department of Radiation Oncology, Kimmel Cancer Center, Jefferson Medical College of Thomas Jefferson University, Philadelphia, USA. 2Department of Radiation Oncology, Lankenau Hospital and Lankenau Institute for Medical Research, Main Line Health System, Pennsylvania, USA. ${ }^{3}$ Department of Medical Oncology, Kimmel Cancer Center, Jefferson Medical College of Thomas Jefferson University, Philadelphia, USA. ${ }^{4}$ Department of Hematology/Oncology, Lankenau Hospital and Lankenau Institute for Medical Research, Main Line Health System, Pennsylvania, USA. ${ }^{5}$ Eli Lilly, Inc., Indianapolis, USA. ${ }^{6}$ Department of Radiation Oncology, Emory University Hospital, Atlanta, USA. 7 Department of Radiation Oncology, University Hospitals, Case Medical Center, Cleveland, USA.

\section{Authors' contributions}

MWW, RA, PG, TM, JT, WJC and MM participated in study design. AD, MWW, WJC and MM participated in the radiation therapy of patients. RA, PG, TM participated in the chemotherapy treatment of patients. XS, AD, and MM participated in data collection. XS and MM performed the data analysis analysis.

All authors read and approved the final manuscript.

\section{Competing interests}

$X S, A D, M W W, R A, P G, T M, W J C, M M$ declare that they have no competing interests.

JT is employed by Eli Lilly, Inc.

Received: 23 September 2010 Accepted: 16 February 2011

Published: 16 February 2011 


\section{References}

1. Curran WJ, Scott CB, Langer CJ, et al: Long-term benefit is observed in a phase III comparison of sequential vs concurrent chemo-radiation for patients with urnsected stage III NSCLC: RTOG 9410. [Abstract]. Proc Am Soc Clin Oncol (ASCO) 2003, 22:621.

2. Fournel $P$, Robinet $G$, Thomas $P$, et al: Randomized phase III trial of sequential chemoradiotherapy compared with concurrent chemoradiotherapy in locally advanced non-small cell lung cancer: Groupe Lyon-Saint-Etienne d'Oncologie Thoracique-Grouope Francais de Pneumocancerologie NPC 95-01. J Clin Oncol 2005, 23:5910-7.

3. Furuse K, Fukuoka M, Kawahara M: Phase III study of concurrent versus sequential thoracic radiotherapy in combination with mitomycin, vindesine, and cisplatin in unresectable stage III non-small cell lung cancer. J Clin Oncol 1999, 17:2692-9.

4. Gandara DR, Chansky K, Albain KS, et al: Long-term survival with concurrent chemoradiation therapy followed by consolidation docetaxel in stage IIIB non-small-cell lung cancer: a phase II Southwest Oncology Group Study (S9504). Clin Lung Cancer 2006, 8:116-21.

5. Belani $\mathrm{CP}$, Choy H, Bonomi $\mathrm{P}$, et al: Combined chemoradiotherapy regimens of paclitaxel and carboplatin for locally advanced non-small cell lung cancer: a randomized phase II locally advanced multi-modality protocol. J Clin Oncol 2005, 23:5883-91.

6. Belani CP, Ramanathan RK: Combined-modality treatment of locally advanced non-small cell lung cancer: incorporation of novel chemotherapeutic agents. Chest 1998, 113:53S-60S.

7. Kim DW, Shyr Y, Shaktour B, Akerley W, Johnson DH, Choy H: Long term follow up and analysis of long term survivors in patients treated with paclitaxel-based concurrent chemo/radiation therapy for locally advanced non-small cell lung cancer. Lung Cancer 2005, 50:235-45.

8. Vokes EE, Herndon J, Kelley MJ, et al: Induction chemotherapy followed by chemoradiotherapy compared with chemoradiotherapy alone for regionally advanced unresectable stage III Non-small-cell lung cancer: Cancer and Leukemia Group B. J Clin Oncol 2007, 25:1698-704.

9. Belani $\mathrm{CP}$, Barstis J, Perry MC, et al: Multicenter, randomized trial for stage IIIB or IV non-small cell lung cancer using weekly paclitaxel and carboplatin followed by maintenance weekly paclitaxel or observation. $\int$ Clin Oncol 2003, 21:2933-9.

10. Socinski MA, Blackstock AW, Bogart JA, et al: Randomized phase II trial of induction chemotherapy followed by concurrent chemotherapy and dose-escalated thoracic conformal radiotherapy (74 Gy) in sage III nonsmall cell lung cancer: CALGB 30105. J Clin Oncol 2008, 26:2457-63.

11. Cohen MH, Johnson JR, Wang YC, Sridhara R, Pazdur R: FDA drug approval summary: pemetrexed for injection (Alimta) for the treatment of nonsmall cell lung cancer. Oncologist 2005, 10:363-8.

12. Zinner RG, Fossella FV, Herbst RS: Pemetrexed in advanced NSCLC: a review of the clinical data. Oncology 2004, 18:54-62.

13. Seiwert TY, Connell PP, Mauer AM, et al: A phase I study of pemetrexed, carboplatin, and concurrent radiotherapy in patients with locally advanced or metastatic non-small cell lung or esophageal cancer. Clin Cancer Res 2007, 13:515-22

14. Bischof M, Weber KJ, Blatter J, Wannenmacher M, Latz D: Interaction of pemetrexed disodium (ALIMTA, multitargeted antifolate) and irradiation in vitro. Int J Radiat Oncol Biol Phys 2002, 52:1381-8.

15. Teicher BA, Chen V, Shih $C$, et al: Treatment regimens including the multitargeted antifolate LY231514 in human tumor xenografts. Clin Cancer Res 2000, 6:1016-23.

16. Hanna N, Shepherd FA, Fossella FV, et al: Randomized phase III trial of pemetrexed versus docetaxel in patients with non-small-cell lung cancer previously treated with chemotherapy. J Clin Oncol 2004, 22:1589-97.

17. Scagliotti GV, Parikh P, von Pawel J, et al: Phase III study comparing cisplatin plus gemcitabine with cisplatin plus pemetrexed in chemotherapy-naive patients with advanced-stage non-small-cell lung cancer. J Clin Oncol 2008, 26:3543-51.

18. Govindan R, Bogart J, Wang X, et al: Phase II study of pemetrexed, carboplatin, and thoracic radiation with or without cetuximab in patients with locally advanced unresectable non-small cell lung cancer: CALGB 30407. [Abstract]. J Clin Oncol 2009, 27:7505.

19. Citron ML, Berry DA, Cirrincione C, et al: Randomized trial of dose-dense versus conventionally scheduled and sequential versus concurrent combination chemotherapy as postoperative adjuvant treatment of node-positve primary breast cancer: first report of Intergroup Trial
C9741/Cancer and Leukemia Group B Trial 9741. J Clin Oncol 2003, 21:1431-9.

20. Kummel S, Krocker J, Kohls A, et al: Randomised trial: survival benefit and safety of adjuvant dose-dense chemotherapy for node-positive breast cancer. Br J Cancer 2006, 94:1237-44.

21. Pfreundschuh M, Trumper L, Kloess M, et al: Two-weekly or 3-weekly CHOP chemotherapy with or without etoposide for the treatment of young patients with good-prognosis (normal LDH) aggressive lymphomas: results of the NHL-B1 trial of the DSHNHL. Blood 2004, 104:626-33.

22. Isonishi S, Yasuda M, Takahashi F, et al: Randomized phase III trial of conventional paclitaxel and carboplatin (c-TC) versus dose-dense weekly paclitaxel and carboplatin (dd-TC) in women with advanced epithelial ovarian, fallopian tube, or primary peritoneal cancer: Japanese Gynecologic Oncology. [Abstract]. J Clin Oncol 2008, 26s:5506.

23. Choy H, Swann S, Walter C, Whipple G, Demas W, Ettinger D: A Phase I trial of Gemcitabine, Carboplatin or Gemcitabine, Paclitaxel and Concurrent Radiation Therapy Followed by Consolidative Gemcitabine and Carboplatin for Inoperable Stage III Non-Small Cell Lung Cancer: An RTOG Study. [Abstract]. J Clin Oncol 2005, 23s:646s.

24. Werner-Wasik M, Swann S, Curran W Jr: A Phase II Study of Cetuximab (C225) In Combination with Chemoradiation (CRT) in Patients (PTS) with Stage IIIA/B Non-Small Cell Lung Cancer (NSCLC): An Interim Overall Toxicity Report of the RTOG 0324 Trial. [Abstract]. J Clin Oncol 2005, 23s:654s.

25. Bradley J, Graham MV, Winter K, et al: Toxicity and outcome results of RTOG 9311: a phase I-II dose-escalation study using three-dimensional conformal radiotherapy in patients with inoperable non-small-cell lung carcinoma. Int I Radiat Oncol Biol Phys 2005, 61:318-28.

26. Yuan S, Sun X, Li M, et al: A randomized study of involved field irradiation versus elective nodal irradiation in combination with concurrent chemotherapy for inoperable stage III nonsmall cell lung cancer. Am J Clin Oncol 2007, 30:239-44.

27. Cullen $\mathrm{MH}$, Zatloukal $\mathrm{P}$, Sorenson $\mathrm{S}$, et al: A randomized phase III trial comparing standard and high-dose pemetrexed as second-line treatment in patients with locally advanced or metastatic non-small-cell lung cancer. Ann Oncol 2008, 19:939-45.

doi:10.1186/1748-717X-6-17

Cite this article as: Shen et al.: Phase i study of 'dose-dense' pemetrexed plus carboplatin/radiotherapy for locally advanced nonsmall cell lung carcinoma. Radiation Oncology 2011 6:17.

\section{Submit your next manuscript to BioMed Central and take full advantage of:}

- Convenient online submission

- Thorough peer review

- No space constraints or color figure charges

- Immediate publication on acceptance

- Inclusion in PubMed, CAS, Scopus and Google Scholar

- Research which is freely available for redistribution

Submit your manuscript at www.biomedcentral.com/submit
C Biomed Central 